\section{Comments And Responses}

\section{Dialogue on Geriatrics: How Should We Fix the Problem?}

TO THE EDITOR: Golden and colleagues (1) ask if geriatric medicine is terminally ill and make a case to restructure training by reallocating resources from fellowship training to teaching initiatives to "gerontologize" medical students and nongeriatricians.

We would counter that geriatrics is very much alive and that the fellowship-trained geriatricians have accomplished much of what Golden and colleagues propose. Academic geriatrics was started in recent decades by nongeriatricians who saw a need to improve the health care of seniors through research, education, and program innovation. They developed fellowship programs to train physicians to lead this movement. The care of seniors has been enriched by this growing number of fellowship-trained geriatricians.

Two points exemplify these accomplishments. First, the geriatrization of students and nongeriatricians has been ongoing for years with support of foundations, the U.S. Department of Veterans Affairs, and the National Institute on Aging and the National Institutes of Health. The impact of geriatricians has been magnified greatly by training many nongeriatrician scholars who have won career development awards in geriatrics, such as those named for the late leaders Dennis W. Jahnigen, T. Franklin Williams, and Paul B. Beeson. The emerging nongeriatricians represent nearly every specialty and subspecialty of medicine and surgery. They would admit that their careers were greatly nurtured by fellowship-trained geriatricians.

Second, new knowledge in caring for seniors is vital and has been accomplished mostly by fellowship-trained geriatricians. For example, consider advances in care delivery systems (such as Guided Care, Hospital at Home, post-hospital care management, acute care of the elderly units, innovations involved in transitions of care) and the understanding, impact, evaluation, prevention, and treatment of polypharmacy, frailty, incontinence, falls, and delirium. Decreasing support of fellowship training and subsequently diminishing the core group of teachers and researchers trained in and focused on geriatrics as suggested by Golden and colleagues would be unwise in light of the increasing number of seniors with complex medical problems coupled with the need for experts trained to guide clinical program development for these individuals.

Nongeriatricians can and should continue to teach geriatric principles and do innovative research to improve the care of seniors. But there remains a need for a robust corps of fellowship-trained geriatricians who stay tightly focused on the care of seniors and who nurture, challenge, and inspire each other and other physicians. The enduring metaphor, "Don't throw the baby out with the bathwater," seems applicable to the Golden and colleagues' thesis.

\section{John R. Burton, $M D$}

Samuel C. Durso, $M D, M B A$

The Johns Hopkins University School of Medicine, Johns Hopkins Bayview Medical Center

Baltimore, MD 21224

Potential Conflicts of Interest: Dr. Burton: Grants/grants pending (money to institution): HRSA Geriatric Education Center, John A. Hartford Foundation (via American Geriatrics Society) for specialists' educa- tion, Donald W. Reynolds Advisory Panel for Geriatric Education Grants; Royalties: JHS Press.

\section{Reference}

1. Golden AG, Silverman MA, Mintzer MJ. Is geriatric medicine terminally ill? Ann Intern Med. 2012;156:654-6. [PMID: 22547474]

TO THE EDITOR: Two recent editorials in Annals cast a very baleful eye on the state of geriatrics in our nation, viewing it either as "terminally ill" (1) or a "societal scotoma" (2). In light of the "graying" of America and the important conceptual clinical practice innovations that geriatrics has developed for comprehensive care of older persons (especially those with multiple and complex "geriatric syndromes"), both articles make clear that the possibility that this specialty may not survive in this nation's health care system is truly a cause for alarm, reconsideration, and corrective action. Indeed, the issues are critical and sufficiently important to call for a multidisciplinary approach to review the current state of geriatrics and make recommendations for its future based on viewpoints from many constituencies-an interaction perhaps best sponsored and supported by a prominent foundation concerned with national health.

I believe that it is also appropriate, as one who has been in a leadership position in academic geriatrics for over 2 decades (3), to indicate a personal point of view here. The establishment of the National Institute on Aging and the appointment of Robert Butler as its founding director in 1974 signaled the potential for emergence of a new medical discipline in this country, one that would define precepts of diversified health care for the elderly, with the underpinning of a growing content of basic biomedical science on aging. From this union, a greater understanding of aging processes and their transitions to chronic diseases could arise (4); be part of clinical practice (5); and form the educational content presented to medical students, trainees, practitioners, and biogerontologists engaged in research (6). In my view, these prospects that would have created a more unified and profound academic discipline have not been achieved: Geriatric practice has not been integrated with its fundamental aging science base. It is still possible to achieve this, however, with the potential for actual "translation" of research studies on aging to ameliorate inception of chronic diseases, a prospect that would enhance the importance of the practice of geriatrics in the coming decades of the 21 st century.

David Hamerman, MD

International Longevity Center

New York, NY 10028

Potential Conflicts of Interest: None disclosed.

\section{References}

1. Golden AG, Silverman MA, Mintzer MJ. Is geriatric medicine terminally ill? Ann Intern Med. 2012;156:654-6. [PMID: 22547474]

2. Leipzig RM, Hall WJ, Fried LP. Treating our societal scotoma: the case for investing in geriatrics, our nation's future, and our patients. Ann Intern Med. 2012;156:657-9. [PMID: 22547475]

3. Hamerman D, Maklan CW. Geriatric practice. Taking up where primary care leaves off. Am J Med. 1987;82:525-8. [PMID: 3826104]

4. Hamerman D. Geriatric Bioscience: The Link Between Aging and Disease. Baltimore: Johns Hopkins Univ Pr; 2007.

5. Hamerman D. Integrating aging into geriatric practice: an emerging orientation for health care [Editorial]. J Am Geriatr Soc. 2010;58:2024-5. [PMID: 20929473] 
6. Hamerman D. Can biogerontologists and geriatricians unite to apply aging science to health care in the decade ahead? J Gerontol A Biol Sci Med Sci. 2010;65:1193-7. [PMID: 20591875]

TO THE EDITOR: Golden and colleagues (1) could rename their In the Balance article "Is the Older Person in America Terminally Ill?"

The essential problem faced by the dearth of geriatricians is not simply, as referred to by the authors, the poor wages paid or the poor reimbursement for caring for elderly patients. Rather, it is the essence of "oldness" of the patients. "Oldness" contributes to turning medical students and physicians away from rendering care to this population.

The many physicians and gerontologists who helped establish the new field of geriatric medicine never intended to have geriatricians render care to the entire population of the very old. The main population served by the field of geriatric medicine is the very old (persons aged 75 years or older). In addition, geriatricians are intended to be teachers of all other physicians-in-training and advisors to health care systems and the government.

Very old persons often have multiple organ abnormalities, including heart disease, gastrointestinal tract issues, and bony skeleton. Many also have memory impairment with a more widespread cognitive defect-the so-called dementia. The skin wrinkles and the general appearance differs greatly from younger years. The less youthful appearance is considered unattractive in our culture and in many others, too.

Golden and colleagues suggest the development of nonphysicians to be able to teach geriatrics or to deliver geriatric care. This is a serious departure from the traditional practice of medicine in the United States. Such radical change would require creation of an officially recognized 2-class system of care, with the continued centrality of physicians in the care of all age populations other than the very old. For that population, our culture would have to embrace the concept that the very old do not deserve to have physicians as their principal medical providers nor as key clinicians on teams developed to render care to them. We have not yet created a computer or a robot that is an effective alternative to a keen physician who is aware and concerned about the very old person's complex clinical history and usually multiple medications_- often averaging 9 medications per day.

It was an innocent error for the field of internal medicine and geriatrics to reduce the fellowship period from 2 years to 1 year. I agree with Golden and colleagues that 1 year is an inadequate length of time for a geriatric specialist to acquire the clinical body of knowledge of geriatrics while also learning to organize, participate, and possibly lead the team necessary to provide enlightened and costeffective care to the very old (2).

The many physicians and gerontologists who contributed to the founding of the field of geriatric medicine did not intend for geriatricians to render care to all of the very old population (about 13 million persons). Rather, the concept of the newly recognized field of geriatric medicine entailed developing leaders who would teach at hospitals, medical schools, and other health care institutions.

It is said that the approach to support and care of the very old is the measure of the tone and ethic of a society. Let those of us within internal medicine not rush to abandon our responsibilities.
Leslie S. Libow, MD

Mount Sinai Medical Center

New York, NY 10025

Potential Conflicts of Interest: None disclosed.

\section{References}

1. Golden AG, Silverman MA, Mintzer MJ. Is geriatric medicine terminally ill? Ann Intern Med. 2012;156:654-6. [PMID: 22547474]

2. Libow LS. Geriatrics in the United States_-baby boomers' boon? N Engl J Med. 2005;352:750-2. [PMID: 15728805]

TO THE EDITOR: The In the Balance article by Golden and colleagues (1) has resulted in a great deal of discussion throughout geriatric medicine programs in Canada. Because geriatric medicine here is a consultative subspecialty as opposed to a primary care discipline, we believe that the situation in Canada is sufficiently different that there is no need to advocate for the "mercy-killing" of Canadian geriatrics. Canadian trainees avidly read Annals and need to be aware that even if this line of reasoning holds in the United States (about which we are dubious), it does not hold in Canada.

In our view, Golden and colleagues have made the error of confusing the Pareto principle with being an expert. The Pareto effect (2) is the well-established phenomenon where $80 \%$ of all problems can be solved with only $20 \%$ of the knowledge base. This is why a well-trained generalist is an essential part of the health care system, because he or she can efficiently and easily deal with $80 \%$ of medical issues. No one in the Canadian health care system claims that all (or even most) older adults should be treated by a geriatrician, any more than all arthritis patients should be cared for by a rheumatologist. Geriatric medicine subspecialists are best utilized by treating the minority of patients with multiple complexities due to frailty-not in internecine squabbles over all patients with graying hair.

Frailty is a highly age-associated state of increasing risk due to an accumulation of deficits that reflect multisystem physiologic changes (3). Firing a shotgun blast of subspecialists to evaluate each organ system independently is both ineffective and expensive, a circumstance remediated by the comprehensive geriatrics assessment (CGA). The most recent meta-analysis showed that the CGA (compared with usual care) reduced patient mortality, increased the chance that a patient would return home, and increased postdischarge cognitive function (4). The research on which the CGA is built requires disciplinary knowledge if it is to be advanced. By not mentioning how the complexity of frailty challenges current models of care, Golden and colleagues miss the entire point of the subspecialty.

There obviously are many issues for Canadian geriatricians to grapple with, such as to what extent they should be involved at the primary care level, the balance between clinical care and research to advance the specialty, and whether more remuneration alone is a fix. We will be addressing these issues in an upcoming issue of the Canadian Geriatrics Journal (5).

Kenneth M. Madden, MD, MSc

University of British Columbia

Vancouver, British Columbia V5Z 1M9, Canada 
Kenneth Rockwood, MD

Dalhousie University

Halifax, Nova Scotia B3H 2E1, Canada

Potential Conflicts of Interest: Dr. Rockwood: Employment: Division of Geriatrics, Faculty of Medicine, Dalhousie University; Other: member of the American Geriatrics Society, the British Geriatrics Society, and the Canadian Geriatrics Society; past-president of the Canadian Society of Geriatric Medicine; serves on the editorial board for several geriatric journals; author on Brocklehurst's Textbook of Geriatric Medicine.

\section{References}

1. Golden AG, Silverman MA, Mintzer MJ. Is geriatric medicine terminally ill? Ann Intern Med. 2012;156:654-6. [PMID: 22547474]

2. Pareto V. Manual of Political Economy. New York: A.M. Kelley; 1971.

3. Rockwood K, Mitnitski A. Frailty defined by deficit accumulation and geriatric medicine defined by frailty. Clin Geriatr Med. 2011;27:17-26. [PMID: 21093719]

4. Ellis G, Whitehead MA, Robinson D, O'Neill D, Langhorne P. Comprehensive geriatric assessment for older adults admitted to hospital: meta-analysis of randomised controlled trials. BMJ. 2011;343:d6553. [PMID: 22034146]

5. Madden KA, Rockwood K. The health of geriatrics in Canada-more than meets the eye. Can Geriatr J. 2012. [Forthcoming]

TO THE EDITOR: The recent In the Balance articles by Golden and colleagues (1) and Leipzig and colleagues (2) invoke much thought. Isn't it premature to declare geriatric medicine terminal rather than address factors for its perceived decline with solutions for healing?

The aging of America warrants development of programs that address functional decline and disability in the elderly (3). The shortage of geriatricians will continue, calling for physician competency in geriatrics (4). Besides internal medicine, several specialties are formulating approaches to include training in geriatric care (4). The American Gastroenterological Association issued a position paper (5) supporting this concept; oncology and other specialties will follow.

The value of geriatric medicine fellowship is questioned (1). As a geriatric medicine fellowship program director for 2 decades and after training about 120 fellows, the value is clearly evident. The differences in approach to geriatric patients by trained geriatricians is obvious in terms of knowledge and confidence, constructing a differential diagnosis, leading a multidisciplinary team, and addressing management (including polypharmacy). One year of training to gain this expertise is merely a fragment of time in a physician's professional life. Most fellows, properly nurtured, enjoy caring for elders and do not regret the year spent. Undoubtedly, geriatrics requires emphasis during internal medicine residency, but how much more can the overworked resident be taught, knowing the regulations imposed by the Accreditation Council for Graduate Medical Education? (6)

Quoting a current fellow in geriatric medicine (second author):

Did I read the Annals article right? I reread in disbelief. Dr. Golden played the devil's advocate well—perhaps a bit too well! Like my trainee colleagues, I tried unsuccessfully to dismiss the negativity that followed. One wonders the impact the article will have on future trainees contemplating geriatrics for a career. The subspecialty gets poor peer recognition in the physician community, lacking a well-defined market niche, possibly from shortage of trained geriatricians. Today, we actually do see older patients seeking geriatricians for care. Additionally, students and residents burdened by huge educational loans might become even more biased against geriatrics on reading these comments, anticipating poor future returns.

Long ago, "pediatrics" was an unrecognized field, and general practitioners treated adults and children alike. Today, it stands distinct and subspecialized. Geriatric medicine is ironically young and needs nurturing to adult years. The Geriatrics Task Force of the New York Chapter of the American College of Physicians, for one, is addressing issues. Pessimism is detrimental. Geriatrics is not a failed experiment but, in fact, a beautiful innovation, young in its life span.

\section{T.S. Dharmarajan, $M D$ \\ Abishek Kumar, MD \\ Montefiore Medical Center \\ Bronx, NY 10466}

Potential Conflicts of Interest: Dr. Dharmarajan: Employment: Vice Chairman of Medicine and Geriatrics Program Director; Payment for lectures including service on speakers bureaus: occasionally paid for CME lectures.

\section{References}

1. Golden AG, Silverman MA, Mintzer MJ. Is geriatric medicine terminally ill? Ann Intern Med. 2012;156:654-6. [PMID: 22547474]

2. Leipzig RM, Hall WJ, Fried LP. Treating our societal scotoma: the case for investing in geriatrics, our nation's future, and our patients. Ann Intern Med. 2012;156:657-9. [PMID: 22547475]

3. Kelley AS, Ettner SL, Morrison RS, Du Q, Sarkisian CA. Disability and decline in physical function associated with hospital use at end of life. J Gen Intern Med. 2012; 27:794-800. [PMID: 22382455]

4. Kuehn BM. Effort under way to prepare physicians to care for growing elderly population. JAMA. 2009;302:727-8. [PMID: 19690295]

5. Hall KE, Proctor DD, Fisher L, Rose S. American Gastroenterological Association future trends committee report: effects of aging of the population on gastroenterology practice, education, and research. Gastroenterology. 2005;129:1305-38. [PMID: 16230084]

6. Nasca TJ, Philibert I, Brigham T, Flynn TC. The next GME accreditation systemrationale and benefits. N Engl J Med. 2012;366:1051-6. [PMID: 22356262]

TO THE EDITOR: The In the Balance articles by Golden and colleagues (1) and Leipzig and colleagues (2) clarified many serious issues related to geriatrics. Nobody knows what lies ahead, but there is one thing that people can accurately forecast in the next few decades: population structure. The declining birth rate and increasing life expectancy push the world, industrialized countries in particular, toward a super-graying society. It is essential in this situation to create a sustainable system that provides better care with lower costs for the elderly. Along with improving reimbursement for geriatricians, increasing the geriatric workforce, and other geriatrics-related issues, attention should be paid to maximizing use of geriatrics specialists.

Identifying clinical niches in which only geriatricians could fill completely is essential. When an earthquake with a magnitude of 9.0 and the subsequent tsunami struck northeast Japan on 11 March 2011, local hospital infrastructure was devastated and countless health records were washed away. Health conditions of many vic- 
tims, elderly people in particular, seriously worsened because of the deterioration of existing chronic conditions, such as heart disease. In this situation, the Japan Geriatrics Society showed a strong presence by distributing about 20000 technical manuals on how to treat elderly patients in the event of a disaster. Moreover, it published handy-to-carry and chart-like notebooks for the elderly so that health care providers clearly understood their chronic conditions and medications. It is worth noting that these notebooks are designed to effectively convey clinical information about age-related risks, such as incontinence, falling, muscle weakness, and difficulty in swallowing. Making up for the declining health literacy of the elderly is undoubtedly one of the crucial roles of geriatricians.

Another important thing is to raise public awareness of when and how geriatricians should be consulted. It is necessary to help the public understand that geriatricians are, as it were, life-planning partners from the viewpoint of patient-centered care (3). The desirable health goals of individual patients tend to change as they age (4). With regard to longitudinal care, access on demand, coordination among subspecialists, and home-based care, geriatricians can play the leading role in treating the elderly with multiple and complex illnesses.

To solve various geriatrics-related issues, the unique characteristics and the importance of geriatrics need to take deep root in society.

\section{Hajime Ichiseki, $M D, P h D$ \\ Joto Ichiseki Clinic \\ Takaoka 933-0041, Japan}

Potential Conflicts of Interest: None disclosed.

\section{References}

1. Golden AG, Silverman MA, Mintzer MJ. Is geriatric medicine terminally ill? Ann Intern Med. 2012;156:654-6. [PMID: 22547474]

2. Leipzig RM, Hall WJ, Fried LP. Treating our societal scotoma: the case for investing in geriatrics, our nation's future, and our patients. Ann Intern Med. 2012;156:657-9. [PMID: 22547475]

3. Reuben DB, Tinetti ME. Goal-oriented patient care—an alternative health outcomes paradigm. N Engl J Med. 2012;366:777-9. [PMID: 22375966]

4. Bardes CL. Defining "patient-centered medicine". N Engl J Med. 2012;366:782-3. [PMID: 22375968]

TO THE EDITOR: We read with great empathy the In the Balance article by Leipzig and colleagues (1) on the dramatic inadequacy of a geriatric workforce facing the increasing number of older persons in the United States. If possible, the state of this crisis is worse in Italy than it is in the United States because we will never be able to reach the required number of geriatricians. (The population of Italy is about one fourth that of the United States, and we hypothesize that we will need about 6500 specialists by the year 2030.) In fact, our academies graduate about 120 geriatricians a year, a rate barely sufficient to balance turnover. Taking into account the economic crisis involving all sectors of our society, it would be unrealistic to plan a swinging increase in the numbers of postgraduate students in this scenario because it would require too much relevant effort of our public budget. For this reason we propose-although with some regret-to concentrate our effort in specific areas, aiming at obtaining results in the short term that are useful for the health of our increasingly older population. First, we should concentrate on setting a number of high-standard research centers dedicated to improve the quality of care from both medical and structural points of view. Obviously, these centers need to be closely linked with hospitals, postacute and rehabilitative settings, nursing homes, and home care services to implement the programs generated by the research. Second, strictly connected with those centers, high-quality teaching programs are needed to influence the education and training of all young physicians (and other professionals) independently from their particular sector of medical interest.

We are well-aware that this is quite a reductive approach, which implicitly might sound like a surrender of the geriatric discipline to demographic and epidemiologic changes. However, following this model, we will probably save the cultural (and hopefully practical) presence of geriatrics in our health care system.

Giuseppe Bellelli, MD

Geriatric Clinic Department of Health Science, University of Milano-Bicocca

Monza, Italy

Marco Trabucchi, MD

Geriatric Research Group

Brescia, Italy

Potential Conflicts of Interest: Dr. Bellelli: Payment for lectures including service on speakers bureaus: Novartis. Dr. Trabucchi: Payment for development of educational presentations: Novartis.

\section{Reference}

1. Leipzig RM, Hall WJ, Fried LP. Treating our societal scotoma: the case for investing in geriatrics, our nation's future, and our patients. Ann Intern Med. 2012;156:657-9. [PMID: 22547475]

IN RESPONSE: Several comments state that our line of reasoning may be "dubious" or "negative." Drs. Burton and Durso even contend that "geriatrics is very much alive" based on teaching and research efforts occurring at academic medical centers. However, no evidence is provided to dispute that the clinical subspecialty of geriatric medicine in the United States remains an unpopular career choice, is poorly reimbursed, and lacks sufficient research demonstrating its effectiveness compared with nongeriatricians (1). In addition, we agree with Dr. Hamerman that despite much progress in the basic science of aging, these efforts have not translated into geriatricianspecific medical interventions.

Dr. Libow discounts poor reimbursement as the major cause for the declining interest in geriatric medicine. Instead, he believes that the negative attitudes of students and physicians toward "oldness" are primarily responsible. Although they are clearly a factor, Dr. Libow undervalues the reality that market forces are a key issue in medicine and human nature. Negative attitudes are often ameliorated by reasonable and equitable incentives. With regard to Dr. Libow's concern about nonphysicians delivering geriatric care, physician assistants and nurse practitioners are already providing geriatric care in outpatient, inpatient, and long-term care settings.

To support the case for geriatrics, Drs. Madden and Rockwood rely on a meta-analysis of CGAs that analyzed data from 22 trials from 6 countries (only 1 study was published during the last 10 years) (2). Two of the studies did not include a geriatrician as part of 
the CGA team. The meta-analysis showed that inpatient interdisciplinary geriatric assessment wards improved clinical outcomes in older adults. Much of the success of these inpatients units was presumed to be based on the specialization of gerontologic nursing and the implementation of multidisciplinary, geriatric-focused protocols (2). The relevance of these data to the effectiveness of geriatricians in the current U.S. health care system remains uncertain (3). In addition, Drs. Madden and Rockwood use the Pareto principle to state that geriatricians should focus their clinical efforts on the care of the frail elderly, yet provide no evidence to explain how limiting geriatric medicine to the care of the frail elderly would revitalize the subspecialty. Focusing on these patients as the clinical niche for geriatricians might further discourage interest in the subspecialty, unless it is accompanied by substantial positive changes in physician recognition and reimbursement. Furthermore, alternative pathways are available for nongeriatricians in the United States to develop the skills needed to care for frail older adults.

There are no simple solutions. Hiding the "negative" aspects of geriatric medicine from medical students and housestaff, as suggested by Drs. Dharmarajan and Kumar, is not the answer. If geriatric medicine is to survive as a clinical subspecialty in the United States, it will require dramatic health care policy reform efforts, some of which are eloquently listed by Leipzig and colleagues (4) and in Dr. Hamerman's comment.

Adam G. Golden, $M D, M B A$

University of Central Florida College of Medicine and Orlando

Veterans Affairs Medical Center

Orlando, FL 32803

\section{Michael A. Silverman, $M D, M P H, C M D$}

West Palm Beach Veterans Affairs Medical Center

West Palm Beach, FL 33410

Michael J. Mintzer, MD

University of Miami Miller School of Medicine and Miami Geriatric

Research Education and Clinical Center, Bruce W. Carter

Veterans Affairs Medical Center

Miami, FL 33125

Potential Conflicts of Interest: Disclosures can be viewed at www .acponline.org/authors/icmje/ConflictOfInterestForms.do?msNum=M11 -3079 .

\section{References}

1. Golden AG, Silverman MA, Mintzer MJ. Is geriatric medicine terminally ill? Ann Intern Med. 2012;156:654-6. [PMID: 22547474]

2. Ellis G, Whitehead MA, Robinson D, O'Neill D, Langhorne P. Comprehensive geriatric assessment for older adults admitted to hospital: meta-analysis of randomised controlled trials. BMJ. 2011;343:d6553. [PMID: 22034146]

3. Totten A, Carson S, Peterson K, Low A, Christensen V, Tiwari A. Evidence Brief: Effect of geriatricians on outcomes of inpatient and outpatient care. VA-ESP Project $\# 09-199 ; 2012$

4. Leipzig RM, Hall WJ, Fried LP. Treating our societal scotoma: the case for investing in geriatrics, our nation's future, and our patients. Ann Intern Med. 2012;156:657-9. [PMID: 22547475]

\section{OBSERVATION}

\section{Antidiuretic Hormone Antagonist to Reduce Cystine Stone Formation}

Background: Cystinuria is the most common inherited cause of recurrent kidney stones (1). Its principal disorder is impaired reabsorption of cystine in the proximal renal tubule. As a result, large

\section{Figure. Changes in urinary cystine concentration during treatment with tolvaptan, $15 \mathrm{mg} / \mathrm{d}$ (shaded areas).}
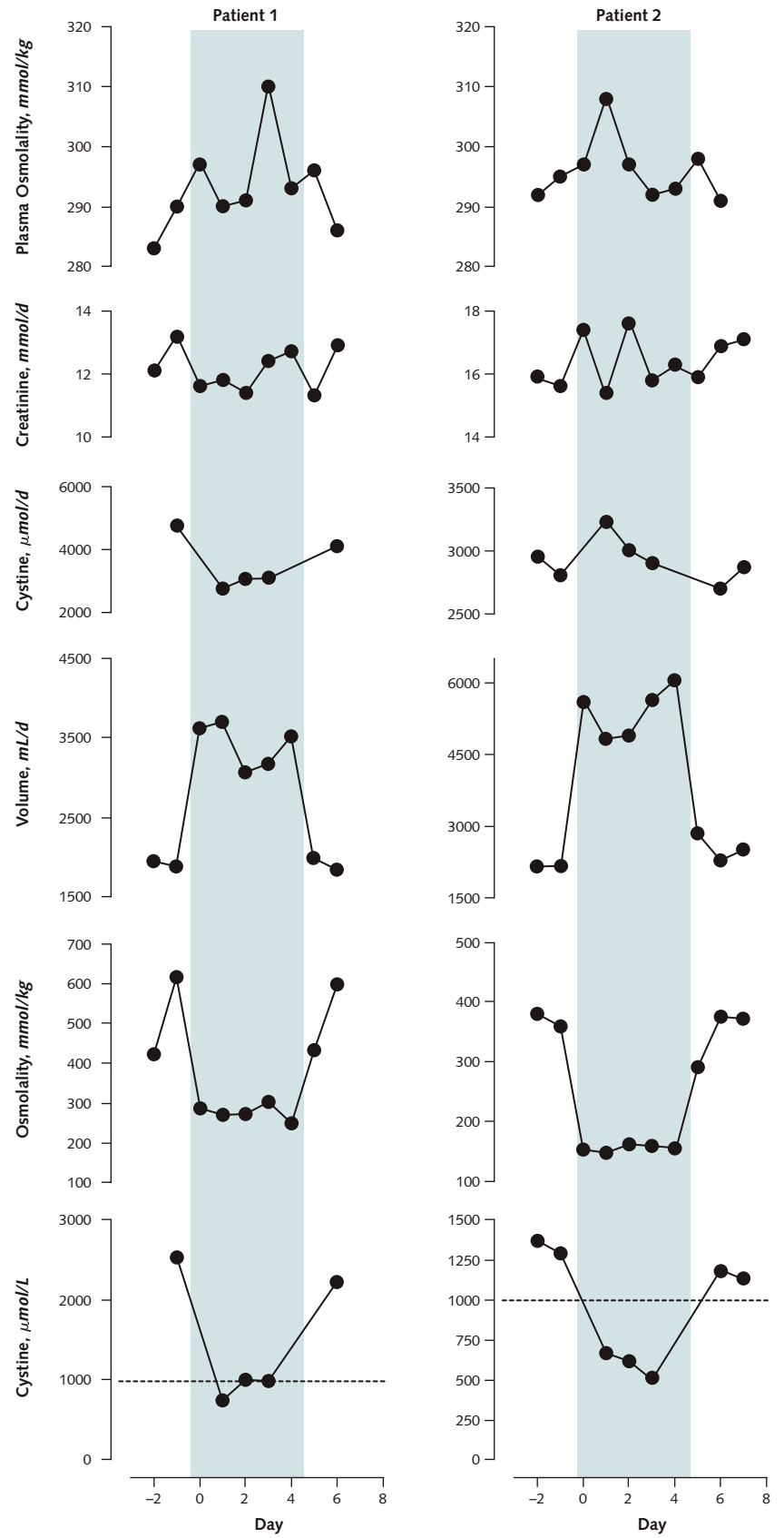
amounts of cystine are excreted in urine, kidney stones form because cystine is poorly soluble, and the patient experiences many urologic interventions. Measures to prevent stone formation include changing the diet to reduce cystine excretion, diluting urine to a lower cystine concentration, and making the urine alkaline to improve cystine solubility. Cystine-binding thiol drugs also improve cystine solubility but need to be discontinued because of adverse effects in $30 \%$ to $60 \%$ of cases (1).

Diluting the urine by increasing oral fluid intake is the key to treatment. The goal is to increase urine volume until the cystine concentration is below $1000 \mu \mathrm{mol} / \mathrm{L}$, its saturation level. In severe cases, this may require a daily fluid intake of more than $3 \mathrm{~L}$. Most patients do not achieve this goal because they are not thirsty, and mental discipline alone is insufficient.

We hypothesized that an antidiuretic hormone antagonist might increase urine volume and reduce cystine concentration below its saturation level $(2,3)$.

Case Reports: Our first patient was a 49-year-old woman with homozygous cystinuria, a nearly normal glomerular filtration rate of $84 \mathrm{~mL} / \mathrm{min}$ per $1.73 \mathrm{~m}^{2}$, and a urinary cystine excretion of about $3500 \mu \mathrm{mol} / \mathrm{d}$ (normal range, 30 to $200 \mu \mathrm{mol} / \mathrm{d}$ ). We advised her to increase water intake and change her diet and prescribed captopril, $50 \mathrm{mg}$ twice daily, but these measures did not decrease urinary cystine concentration. We also prescribed tiopronin, $100 \mathrm{mg}$ three times daily, but had to discontinue it because of severe rash.

Our second patient was a 35-year-old man with homozygous cystinuria, recurrent cystine stones leading to intermittent urinary tract obstruction, glomerular filtration rate of $49 \mathrm{~mL} / \mathrm{min}$ per 1.73 $\mathrm{m}^{2}$, and a urinary cystine excretion rate of about $3000 \mu \mathrm{mol} / \mathrm{d}$.

After obtaining informed consent from both patients, we treated them with tolvaptan, $15 \mathrm{mg}$ once daily for 5 days. Both patients responded (Figure) with a minor increase in plasma osmolality and a substantial increase in daily urine volume that decreased the cystine concentration below $1000 \mu \mathrm{mol} / \mathrm{L}$ without affecting daily cystine excretion. Both patients reported a minor increase in thirst, but neither perceived this as bothersome. Patient 1 did not experience nocturia; patient 2 urinated once each night.

Conclusion: Our experience with these 2 patients suggests that an antidiuretic hormone antagonist may be useful to dilute urine below the cystine supersaturation level, which should prevent formation of new stones and might dissolve existing ones. The approach is not specific for cystinuria but should be applicable to any form of kidney stone that responds to urine dilution. As a result of our experience, we believe it would be worthwhile to conduct a clinical trial that examines the use of antidiuretic hormone antagonists for treating recurrent kidney stones that are resistant to conventional treatment. Such a trial should measure such adverse effects as polyuria, nocturia, and thirst. Researchers should calculate the minimum volume of urine required for each participant to prevent supersaturation, based on baseline daily urine excretion levels and known saturation levels. A successful trial will require researchers to achieve a specific urine volume in each patient and to maintain it for a relatively long period.

Hans de Boer, MD, PhD

Anita Roelofsen, $M D$

Pim M.W. Janssens, PhD

Rijnstate Hospital

6881 NR Arnhem, the Netherlands

Potential Conflicts of Interest: None disclosed.

\section{References}

1. Mattoo A, Goldfarb DS. Cystinuria. Semin Nephrol. 2008;28:181-91. [PMID: 18359399]

2. Decaux G, Soupart A, Vassart G. Non-peptide arginine-vasopressin antagonists: the vaptans. Lancet. 2008;371:1624-32. [PMID: 18468546]

3. Doggrell SA. Tolvaptan (Otsuka). Curr Opin Investig Drugs. 2004;5:977-83. [PMID: 15503654]

\section{Personae Photographs}

Annals of Internal Medicine invites submissions of Personae photographs for our cover and offers a $\$ 500$ prize for the best photograph submitted each year. Personae photographs are pictures that catch people in the context of their lives and that capture personality. We prefer black-andwhite digital files. Please submit photographs or questions to Renee Wise (rwise@acponline.org). 